\title{
A bio-hybrid rotaxane catalyst
}

\author{
Joost Clerx, ${ }^{\dagger,+}$ Stijn F. M. van Dongen, ${ }^{+,+}$Kasper Nørgaard,${ }^{\dagger}$ Michael A. Trakselis,,+ \\ Scott W. Nelson, ${ }^{\S}$ Stephen J. Benkovic, "Alan E. Rowant,* and Roeland J. M. Noltet,*
}

\begin{abstract}
$\dagger$ Radboud University Nijmegen, Institute for Molecules and Materials
Heyendaalseweg 135, 6525 ED, Nijmegen, The Netherlands

t Department of Chemistry, 801 Chevron Science Center,

219 Parkman Avenue, Pittsburgh, PA 15260, United States of America

$\S$ Biochemistry, Biophysics \& Molecular Biology, Iowa State University, Ames, IA 50011, United States of America

\| Department of Chemistry, 414 Wartik Laboratory, The Pennsylvania State University, University Park, Pennsylvania, 16802, United States of America

+ These authors contributed equally to this report
\end{abstract}

Abstract. In processive catalysis, a catalyst binds to a substrate and remains bound while performing several consecutive reactions, as exemplified by DNA polymerases.

Processivity is essential in Nature, and is often mediated by a clamp-like structure that physically tethers the catalyst to its (polymeric) template. In the case of the bacteriophage T4 replisome, a dedicated clamp protein acts as a processivity mediator by encircling DNA and subsequently recruiting its polymerase. We used this DNA-binding clamp to construct a bio-hybrid rotaxane catalyst. To this end, the clamp was conjugated to a chemical catalyst with sequence-specific oxidation behaviour. The resulting catalytic clamp could be loaded onto a DNA plasmid, and its catalytic activity was visualised using an AFM-based method that detects and spatially locates oxidised sites in DNA. It was found that both the catalytic processivity and the sliding direction of the rotaxane catalyst can be influenced by varying experimental conditions.

Toroidal processive enzymes, i.e. enzymes that are able to thread onto biopolymers and to perform stepwise reactions along the polymer chain, are among the most fascinating tools involved in the clockwork machinery of life. Processive catalysis is ubiquitous in Nature and contrasts the nontemplated distributive catalysis, which is the most common mode of operation for both 
homogeneous and heterogenous catalysts. ${ }^{1}$ Examples of processive catalysts are DNA polymerases and exonucleases, which play crucial roles in numerous events during the life cycle of a cell, including most of the replication, transcription, and repair processes..$^{2-7}$ Clamp-shaped proteins are central components in these metabolic processes. They encircle DNA and can tether other proteins or enzymes through specific binding interactions. Clamp proteins can track along DNA, either as a single entity or as an enzyme carrier, and are loaded at recognition sites by clamp loader proteins. Therefore, the clamp enhances the association of its recruited enzyme, conferring processivity to the latter's catalytic action. A well-studied example is the replication system of the bacteriophage T4, which employs a trimeric ring-shaped clamp (gp45) to associate with the replication polymerase (gp43) to DNA, thereby dramatically enhancing the processivity of the latter enzyme. ${ }^{8,9}$ While the clamp itself has no catalytic function, it effectively enables the polymerase to switch from a distributive to a processive mode of action, paramount to the efficiency of the replication process.

In the past, many studies have been reported on synthetic systems that model the catalytic action of enzymes. ${ }^{10-12}$ In contrast, very few efforts have been paid to mimic the processive properties of these biomacromolecules. ${ }^{13}$ In previous papers, we have described a synthetic catalyst based on a macrocyclic porphyrin complex which can thread onto a synthetic polymer chain (polybutadiene) and oxidise its double bonds via a hopping mechanism, ${ }^{14-16}$ thereby acting as a primitive model of a processive enzyme. In the present paper we report on a biohybrid mimic, which is derived from the T4 sliding clamp protein. The mimic is equipped with a porphyrin derivative to yield an artificial enzyme that can oxidise DNA substrates. Depending on the reaction conditions, its mode of catalysis can be influenced from a distributive to a processive mode of action. The oxidation reactions are monitored at the single molecule level using a novel streptavidin labelling procedure 
in combination with atomic force microscopy (AFM), which provides a tool that not only can detect catalytic events, but can also spatially relate them to each other. As a catalyst we used the manganese tetramethyl pyridinium porphyrin complex (Mn-TMPyP, 1, see Fig. 1), which together with an oxygen donor can selectively cleave double stranded DNA (dsDNA) templates at sequences containing three consecutive adenosine-thymine (A-T) base pairs. ${ }^{17-19}$ The main pathway of this oxidation process entails hydroxylation at the $\mathrm{C}^{5}$ ' position of the deoxyribose ring of the DNA backbone, leading to a nick at the 3 ' end of the AAA sequence. This nick contains an aldehyde group at its 5 ' end, which forms a suitable target for the above mentioned streptavidin assay. Combining the activity of this porphyrin with the DNA-tracking behaviour of the T4 clamp protein thus effectively creates a bio-hybrid rotaxane catalyst with a processive character.

\section{Results and discussion}

Design and synthesis of the bio-hybrid rotaxane catalyst. A maleimide-functionalised porphyrin based on Mn-TMPyP (2, see Fig. 1) was synthesised as described in the supplementary information. We used a gp45 E212C mutant of the T4 clamp as a scaffold for conjugation with 2, because it features a conveniently located cysteine residue on each of the protein monomer subunits, which can be reacted with the maleimide moiety of $\mathbf{2}$. The porphyrin catalyst needs to have sufficient flexibility to bind to the minor groove of DNA. To achieve this, the porphyrin moiety was separated from the gp45 clamp by a short water-soluble ethylene glycol segment. The labelling of the gp45 mutant with 2 was carried out with an excess (10 eq.) of the latter compound for 5 hours. Due to the trimeric nature of the clamp and the presence of a single available cysteine group on each subunit, the resulting gp45-2 conjugate can contain up to three porphyrin catalysts. Anion-exchange chromatography allowed for the separation of the different compounds containing 1, 2 or 3 
porphyrins per trimer. The species with the highest porphyrin content was used for experiments described in this report, and will hereafter be called 'catalytic clamp.'

Additionally, we synthesised an octapeptide (3, see Fig. 1) with a sequence similar to the Cterminus of the T4 polymerase (gp43). ${ }^{20}$ This peptide simulates the effect of gp43, leading to clamp loader departure and effective closure of the gp45 clamp on DNA. Alternatively, the peptide may complete the ring structure of the clamp by binding in the open subunit interface, blocking access by the clamp loader and preventing association of the clamp with DNA substrates.

Interaction of the clamp protein with DNA. Two methods exist to allow gp45 to associate with DNA: it can either be assembled onto DNA via nonspecific binding, or it can be loaded by a specific T4 clamp loader protein complex (gp44/62). ${ }^{21,22}$ Both techniques have been used to successfully load the clamp onto various DNA substrates. The latter requires the hydrolysis of ATP and a suitable DNA template containing a primer-template site. This gp44/62-mediated loading process can be monitored using an enzyme-coupled ATPase assay. ${ }^{20}$ We used this assay to investigate the interaction between octapeptide $\mathbf{3}$ and the clamp protein. Using a small forked DNA substrate, ${ }^{22}$ we found that the ATP hydrolysis rate decreased when the octapeptide was titrated into a solution containing the DNA, gp45 and the clamp loader complex (see supplementary information, Fig. S7). This indicates that less successful loading events take place, suggesting the above alternate explanation, where the peptide inhibits clamp loading, applies.

AFM as a tool for detecting the oxidation of DNA. We performed oxidation experiments with the labelled clamp on supercoiled DNA plasmid substrates consisting of 3540 base-pairs and based on a commercially available plasmid (pGEM, see supplementary information). The supercoiled form of the plasmid (Form I) provides a sensitive method to probe for oxidation events: introduction of a 
single stranded break results in uncoiling of the plasmid to the relaxed circular form (Form II). ${ }^{23}$ This transition is easily monitored by conventional agarose gel electrophoresis, owing to the large difference in relative mobility of the two species. Further oxidation events only result in a difference in mobility when a double stranded break occurs and the plasmid is converted to the linear form (Form III), which requires two oxidation events on opposite strands that are no more than 16 nucleotides apart, ${ }^{24}$ which is a rare occurrence due to the sequence specificity of our catalyst. Consequently, gel electrophoresis is not a suitable technique if detailed analysis of plasmid oxidations is needed. Furthermore, if nicked DNA is used as starting material, gel electrophoresis cannot detect any oxidations that do not lead to a double strand break.

Since we required a more sensitive oxidation assay, we looked into alternative ways of analysing the reaction products. The group of Meunier has shown that the aldehydes in porphyrin-oxidised DNA oligonucleotides are available for chemical modifications such as reductive amination. ${ }^{25}$ An aldehyde reactive probe (ARP, 4, see Fig. 1) based on hydroxylamine-derived biotin was used to allow a more detailed investigation of the oxidised sites. Through the interaction between biotin and streptavidin, oxidised sites in the plasmid can be marked by a protein, which is a large structural feature compared to the DNA strand (streptavidin has a molecular weight of $\sim 60 \mathrm{kDa}$ and an apparent height of $\sim 4.4 \mathrm{~nm}$, as compared to a typical height of $\sim 1.2 \mathrm{~nm}$ for dsDNA). AFM can be used to identify these protein labels. ${ }^{26}$ Here, we identify streptavidin to determine the number of oxidation events, and to elucidate their spatial distribution. A schematic representation of this strategy is presented in Fig. 2a. In the fast-growing body of literature describing DNA oxidation by porphyrins, the aldehyde has not been used for the quantisation of oxidation damage. Moreover, information regarding the spatial distribution of the reactions, to detect processivity in substrate oxidation, could hitherto not be obtained because a suitable assay was not available. 
To validate this technique, we incubated DNA with Mn-TMPyP 1 in the presence of $\mathrm{KHSO}_{5}$ as an oxygen donor. After one minute, the reaction was quenched and ARP was added to biotinylate any oxidised sites. The purified solution was supplemented with a streptavidin solution to achieve protein labelling of reaction sites, after which it was analysed by AFM. Figure $2 \mathrm{~b}$ shows a representative AFM height image displaying two relaxed circular DNA strands with a number of globular features. A profile analysis of this image is given in Fig. 2c. The globular features have apparent heights ranging from 3.3 to $5.4 \mathrm{~nm}$, which is in agreement with the height measured for streptavidin $(\sim 4.4 \mathrm{~nm})$. This experiment demonstrated that the combination of ARP, streptavidin and AFM provides an alternative tool for assaying DNA oxidation by Mn-porphyrins such as $\mathbf{1}$.

\section{Influence of the octapeptide on DNA oxidation by the catalytic clamp. Supercoiled DNA}

plasmid was incubated with the catalytic clamp and $\mathrm{KHSO}_{5}$ as an oxygen donor, with or without octapeptide 3 present. Gel electrophoresis confirmed that nearly all supercoiled DNA was converted into the nicked form (see Fig. 3a). As expected, the addition of peptide resulted in a decrease in oxidation efficiency: the binding of the clamp protein to DNA is inhibited by the binding of the peptide to the open subunit interface of a clamp trimer. This suggests that the peptide acts like a switch, reminiscent of the peptide switch in the E. coli replication mechanism that mediates processivity by regulating polymerase association with DNA. ${ }^{6}$ Plasmids treated with the incubation mixture for one minute were analysed by AFM, and the number of oxidation events per plasmid was recorded for conditions with or without the octapeptide present. Without the peptide, $80 \pm 5 \%$ of the plasmids were found to be oxidised in this timespan. Oxidised plasmids were observed to have an average amount of 2.3 bound streptavidin molecules. The histogram shown in Fig. $3 \mathrm{~b}$ appears to be constituted of two Poissonian curves with maxima at 1 or at 5 oxidation events per plasmid. Individual analysis of plasmids from the latter subset revealed that bound streptavidin molecules 
were often found in clusters (see Fig. 3c). If only distributive oxidation would have taken place, these dots would have been distributed over the substrate. Therefore, the clusters might be a tentative indication of processive oxidation by the catalytic clamp. If so, the amount of processive oxidation is still relatively small, namely around $17 \%$. In any case, no such processive clusters were found in experiments where octapeptide $\mathbf{3}$ was present, and less oxidation events took place overall: $55 \pm 5 \%$ of the plasmids were oxidised, and no more than three streptavidin labels were found on any single plasmid (see Fig. 3c, bottom panel). This reduced catalytic activity suggests that the clamp has a lower affinity for DNA when $\mathbf{3}$ is present, corroborating the finding that $\mathbf{3}$ effectively closes the clamp (see Fig. 3d and Fig. S7).

The direction of the catalytic clamp can be guided. In Nature, the loading of the gp 45 clamp protein onto its DNA substrate is mediated by the clamp loader complex (gp44/62) in an ATPdependent process. ${ }^{27}$ First, the clamp loader complex associates with the gp45 clamp protein. Subsequently, the clamp is loaded onto DNA at a preferential binding site. These sites can vary from simple nicks in dsDNA to a 5' extended single overhang. ${ }^{28}$ When a nick is used as a loading site, the clamp loader complex binds to the 3' end of the nick, and it remains bound while the clamp itself is free to slide over the DNA. Thus, the nick effectively determines the direction in which the clamp will translocate, because one of the two possible paths is physically blocked by the gp44/62 complex (See Fig. 4a).

Nicks in plasmids can be created with exact precision by engineered restriction enzymes. ${ }^{29}$ Wild type restriction enzymes are nucleases which recognise and cut fixed DNA sequences, causing double stranded breaks. One such restriction enzyme is BbvCI. Its recognition sequence and cut sites are shown in Fig. 4b. Two engineered versions of BbvCI that only cut one of the two strands of 
the recognition site exist. These two mutants, Nt.BbvCI and Nb.BbvCI, do not cause doublestranded breaks and thus effectively only produce nicks in one of the two strands. ${ }^{29}$ Their cut sites are also shown in Fig. 4b. We were interested in whether nicks generated by one of these engineered restriction enzymes could in effect create loading sites that, through their predetermined interaction with the clamp loader complex, specifically direct the catalytic clamp in either the 3 ' or the 5' direction of the plasmid. We selected our pGEM-derived plasmid as template because it conveniently contains only a single recognition site for BbvCI, which lies in between two regions that have either low or high AAA site densities (see Fig. 4c). If $\mathrm{Nb} . \mathrm{BbvCI}$ is used to nick the plasmid, the catalytic clamp should be directed towards a region sparse in potential oxidation sites, whereas a nick caused by Nt.BbvCI should direct the plasmid towards a region with many potential oxidation sites.

To verify this, the plasmid was nicked with either the Nt.BbvCI or the $\mathrm{Nb} . \mathrm{BbvCI}$ endonuclease, and the catalytic clamp was loaded by the clamp loader. Streptavidin labelling analysis was successful in identifying oxidation reactions on the nicked substrates. As can be seen in Figs. 5a and 5b., the different effects of the two BbvCI variants is immediately apparent; when the Nt.BbvCI-nicked substrate was used, the average number of streptavidin molecules per plasmid was $6.1 \pm 1.6$. For the Nb.BbvCI substrate, this number was significantly lower, namely $1.9 \pm 1.2$ (see Fig. 5c). This observation correlates with the situation outlined in Fig. 4, where the clamp is loaded and subsequently migrates towards regions with either a high or a low amount of oxidation sites. Moreover, visual inspection of the location of streptavidin labels on Nt.BbvCI-nicked substrates revealed that oxidations consistently occurred in clusters. To reach the first cluster of AAA sites in the 5 ' direction of the loading site, the catalytic clamp would have to slide across more than 40 base pairs on the substrate, clearly demonstrating the processive nature of this hybrid catalyst. 


\section{Conclusion}

We have constructed a novel bio-hybrid artificial enzyme based on the T4 clamp protein and a conjugated cationic manganese porphyrin catalyst. The resulting catalytic clamp is capable of translocating along a DNA template and oxidising AAA sites. To analyse its activity, we developed an AFM-based method in which oxidised sites on the plasmid are labelled with streptavidin. Identification of the streptavidin-labels on the DNA substrate by AFM was not only successful in detecting oxidation events, but also in gathering information about their relative location, which hitherto had not been possible.

Depending on the reaction conditions, the biohybrid catalyst displayed either distributive or processive catalytic behaviour on DNA substrates. In cases where the protein was bound to DNA through nonspecific binding, clusters of streptavidin labels in close proximity could be observed on the same plasmid, implying processive catalysis. Such clusters were not found when the catalytic clamp was 'closed' by an octapeptide $\mathbf{3}$, a structural mimic of the sequence that naturally completes the circular shape of the clamp. In this closed state, the catalytic clamp could not associate with its DNA template and thus all reaction products were the result of distributive actions.

In Nature, the T4 clamp protein is loaded onto recognition sites in DNA by a clamp loader complex (gp44/62). The orientation of the recognition site determines the direction in which the clamp will slide along the DNA template. We could enzymatically generate two mirrored loading sites, guiding the clamp to stretches of DNA that were either rich or poor in potential reaction sites. The unique ability of our AFM-method for detecting catalytic events allowed us to verify that when this directed enzymatic loading strategy was applied, the catalyst would indeed slide towards the chosen region. If the catalytic clamp was guided towards the substrate-rich region, we could detect many 
streptavidin labels appearing in clusters. When the clamp slid over the substrate-poor region, a lower total amount of oxidation events was found, and no clusters of two or more labels were detected.

Combined, this report shows that using concepts from Nature, such as toroidal mediators, the performance of catalytic reactions may be influenced and guided, allowing the development of more efficient lifelike catalysts for the modification of linear substrates, eventually also synthetic polymers.

\section{References}

1. Breyer W. A. \& Matthews B. W. A structural basis for processivity. Protein Sci. 10, 1699-1711 (2001).

2. Stillman B. Smart machines at the DNA-replication fork. Cell 78, 725-728 (1994).

3. Kovall R. \& Matthews B. W. Toroidal structure of $\lambda$-exonuclease. Science 277, 1824-1827 (1997).

4. Benkovic S. J., Valentine A. M., \& Salinas F. Replisome-mediated DNA replication. Annu. Rev. Biochem. 70, 181-208 (2001).

5. Champoux J. J. DNA topoisomerases: structure, function, and mechanism. Annu. Rev. Biochem. 70, 369-413 (2001).

6. López de Saro F. J., Georgescu R. E., \& O'Donnell M. A peptide switch regulates DNA polymerase processivity. Proc. Natl. Acad. Sci. U. S. A. 100, 14689-14694 (2003). 
7. Indiani C., McInerney P., Georgescu R., Goodman M. F., \& Oí Donnell M. A sliding-clamp toolbelt binds high-and low-fidelity DNA polymerases simultaneously. Mol. Cell 19, 805-815 (2005).

8. Trakselis M. A., Alley S. C., Abel-Santos E., \& Benkovic S. J. Creating a dynamic picture of the sliding clamp during T4 DNA polymerase holoenzyme assembly by using fluorescence resonance energy transfer. Proc. Natl. Acad. Sci. U. S. A. 98, 8368-8375 (2001).

9. Yang J., Zhuang Z., Roccasecca R. M., Trakselis M. A., \& Benkovic S. J. The dynamic processivity of the T4 DNA polymerase during replication. Proc. Natl. Acad. Sci. U. S. A. 101, 8289-8294 (2004).

10. Breslow R. Artificial enzymes. Science 218, 532-537 (1982).

11. Murakami Y., Kikuchi J., Hisaeda Y., \& Hayashida O. Artificial enzymes. Chem. Rev. 96, $721-758(1996)$.

12. Nanda V. \& Koder R. L. Designing artificial enzymes by intuition and computation. Nat. Chem. 2, 15-24 (2010).

13. Takashima Y., Osaki M., Ishimaru Y., Yamaguchi H., \& Harada A. Artificial molecular clamp: a novel device for synthetic polymerases. Angew. Chem., Int. Ed. 50, 7524-7528 (2011).

14. Thordarson P., Bijsterveld E. J., Rowan A. E., \& Nolte R. J. Epoxidation of polybutadiene by a topologically linked catalyst. Nature 424, 915-918 (2003).

15. Coumans R. G. E., Elemans J., Nolte R. J. M., \& Rowan A. E. Processive enzyme mimic: kinetics and thermodynamics of the threading and sliding process. Proc. Natl. Acad. Sci. U. S. A. 103, 19647-19651 (2006). 
16. Monnereau C., Ramos P. H., Deutman A. B. C., Elemans J., Nolte R. J. M., \& Rowan A. E. Porphyrin macrocyclic catalysts for the processive oxidation of polymer substrates. J. Am. Chem. Soc. 132, 1529-152+ (2010).

17. Meunier B. Metalloporphyrins as versatile catalysts for oxidation reactions and oxidative DNA cleavage. Chem. Rev. 92, 1411-1456 (1992).

18. Pratviel G., Bernadou J., \& Meunier B. DNA and RNA cleavage by metal complexes. $A d v$. Inorg. Chem. 45, 251-312 (1998).

19. Bernadou J. \& Meunier B. Biomimetic chemical catalysts in the oxidative activation of drugs. Adv. Synth. Catal. 346, 171-184 (2004).

20. Berdis A., Soumillion P., \& Benkovic S. The carboxyl terminus of the bacteriophage T4 DNA polymerase is required for holoenzyme complex formation. Proc. Natl. Acad. Sci. U. S. A. 93, 12822-12827 (1996).

21. Zhuang Z. H., Berdis A. J., \& Benkovic S. J. An alternative clamp loading pathway via the T4 clamp loader gp44/62-DNA complex. Biochemistry 45, 7976-7989 (2006).

22. Smiley R. D., Zhuang Z. H., Benkovic S. J., \& Hammes G. G. Single-molecule investigation of the T4 bacteriophage DNA polymerase holoenzyme: Multiple pathways of holoenzyme formation. 45, 7990-7997 (2006).

23. Vologodskii A. V., Lukashin A. V., Anshelevich V. V., \& Frank-Kamenetskii M. D. Fluctuations in superhelical DNA. Nucleic acids res. 6, 967-982 (1979).

24. Freifeld D. \& Trumbo B. Matching of single-strand breaks to form double-strand breaks in DNA. Biopolymers 7, 681-68\& (1969). 
25. Pratviel G., Duarte V., Bernadou J., \& Meunier B. Nonenzymic cleavage and ligation of DNA at a three A $\bullet$ T base pair site. A two-step pseudohydrolysis of DNA. J. Am. Chem. Soc. 115, 7939-7943 (1993).

26. Neish C. S., Martin I. L., Henderson R. M., \& Edwardson J. M. Direct visualization of ligandprotein interactions using atomic force microscopy. Br. J. Pharmacol. 135, 1943-1950 (2002).

27. Alley S. C., Abel-Santos E., \& Benkovic S. J. Tracking sliding clamp opening and closing during bacteriophage T4 DNA polymerase holoenzyme assembly. Biochemistry 39, 3076-3090 (2000).

28. Jarvis T. C., Paul L. S., Hockensmith J. W., \& von Hippel P. H. Structural and enzymatic studies of the T4 DNA replication system. II. ATPase properties of the polymerase accessory protein complex. J. Biol. Chem. 264, 12717-12729 (1989).

29. Heiter D. F., Lunnen K. D., \& Wilson G. G. Site-specific DNA-nicking mutants of the heterodimeric restriction endonuclease R.BbvCI. J. Mol. Biol. 348, 631-640 (2005).

\section{Acknowledgements}

We thank Michelle M. Spiering and Zhihao Zhuang for their assistance and for useful discussions.

We thank Maria Blasco Castells and Dennis van de Mosselaar for synthetic assistance, and Tom G. Bloemberg for insightful comments. This work was supported by the Royal Netherlands Academy of Arts and Sciences (R.J.M.N.), NWO Vici (A.E.R.) and a research grant from the European Research Council (ERC-2011-AdG 290886 ALPROS, to S.F.M.v.D. and R.J.M.N.).

\section{Author Contributions}

S.J.B. and R.J.M.N. conceived the experiments; A.E.R., R.J.M.N. and J.C. designed the 
experiments. J.C. performed the experimental work; K.N. developed the AFM-analysis and M.A.T. and S.W.N. provided expertise on protein design and expression; S.F.M.v.D. performed additional experimental work; J.C., S.F.M.v.D., K.N., A.E.R. and R.J.M.N. analysed and interpreted the results. S.F.M.v.D. and J. C. wrote the manuscript and all authors commented on it.

\section{Additional information}

The authors declare no competing financial interests. Supplementary information and chemical compound information accompany this paper at www.nature.com/naturechemistry. Reprints and permission information is available online at http://npg.nature.com/reprintsandpermissions/. Correspondence and requests for materials should be addressed to A.E.R. and R.J.M.N. 
a
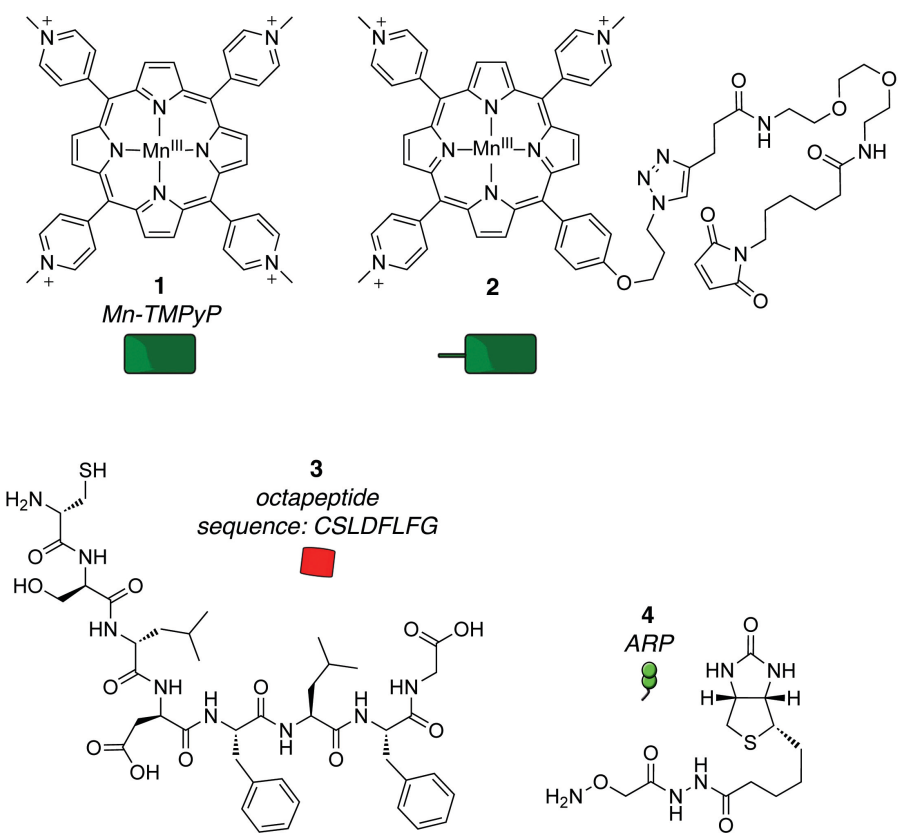

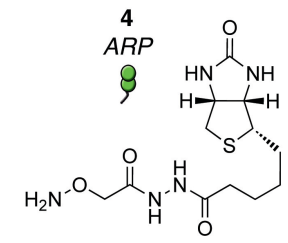

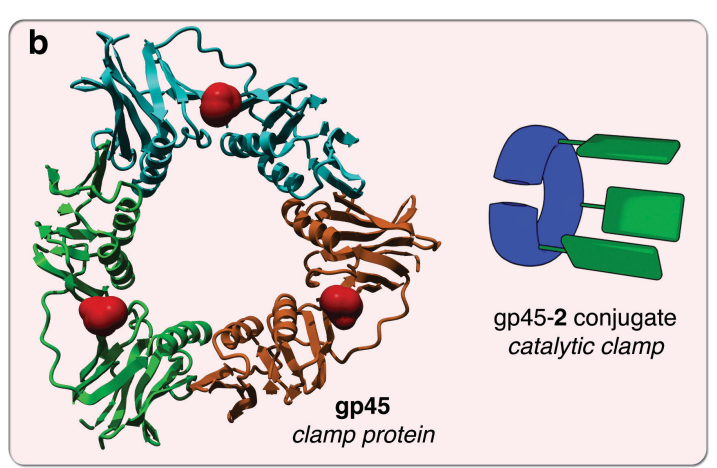

C

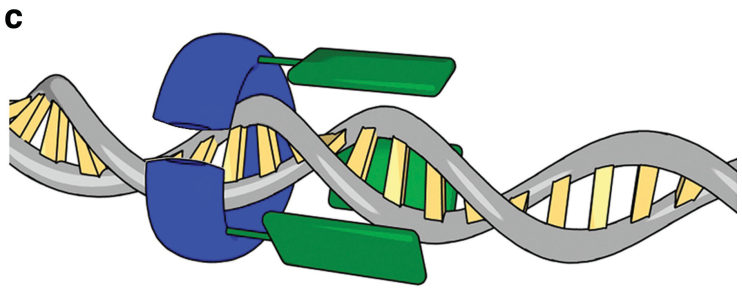

Figure 1 I Schematic representations of structures and concepts. a, Structural formulae, colloquial names and cartoon representations of small molecules. b, Cartoon representations and colloquial names of proteins and protein conjugates. The E212C mutant of gp45 is shown, and its cysteine residues are emphasised in red. Its structure is based on pdb-file 1CZD with the E212C mutation performed in silico. c, The concept for a bio-hybrid rotaxane catalyst is based on synthetic catalyst 2 , which is conjugated to the gp45 clamp protein to form a catalytic clamp. This bio-hybrid catalyst can associate with DNA and slide along it, conferring processivity to the catalytic behaviour of 2. 
a

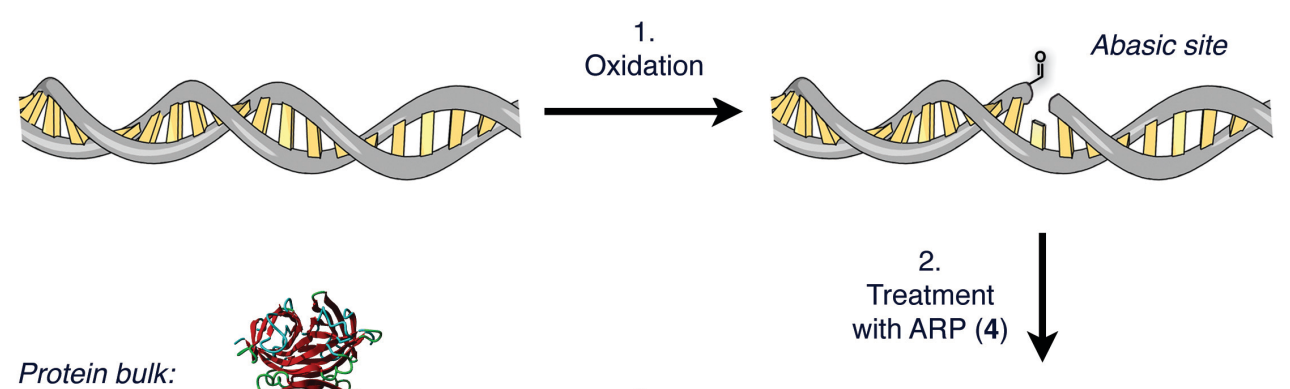
observable by $A F M$

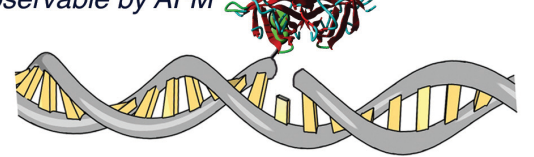

3.

Streptavidin 9 Biotinylated site labelling

b

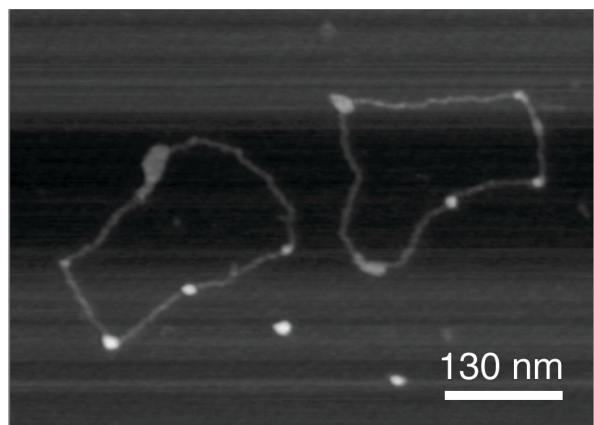

C

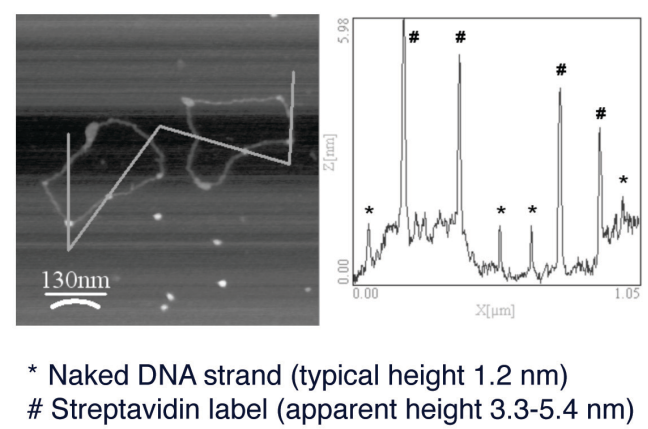

Figure 2 I AFM-based method for detecting DNA oxidation. a, Schematic

representation of the detection strategy: 1) DNA oxidation produces an aldehyde; 2) The aldehyde reacts with the ARP reagent 4 to produce a biotinylated site; 3) Treatment with streptavidin results in a large-sized object that is connected to the oxidation site, which can be detected by AFM. b, Typical AFM height image showing two relaxed circular DNA plasmids with a number of globular features. c, Profile analysis following the indicated line in the left panel. Different features are indicated as being a naked DNA strand $\left(^{*}\right)$ or a DNA strand with a globular feature (\#). Streptavidin has an apparent height of $\sim 4.4 \mathrm{~nm}$, which corresponds to the height of the globular features labelled "\#”. 


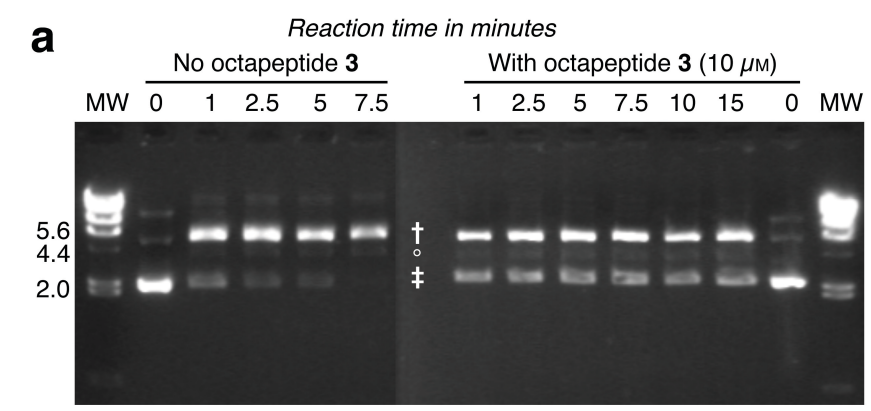

C
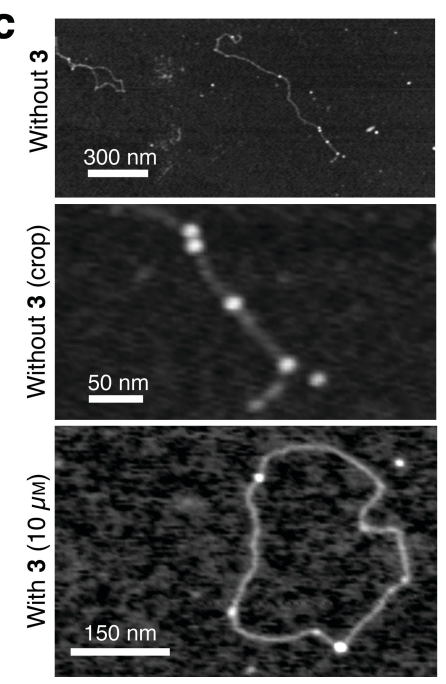

d

"Open" catalyic clamp
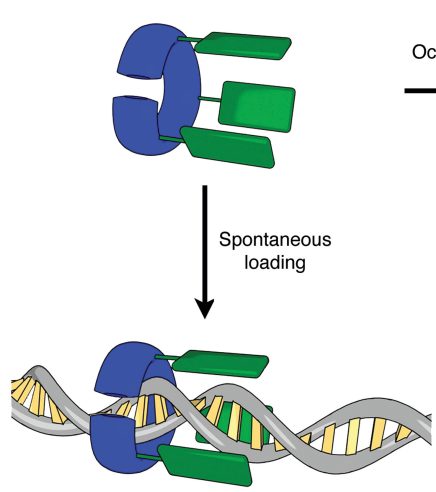

Processive catalysis possible b
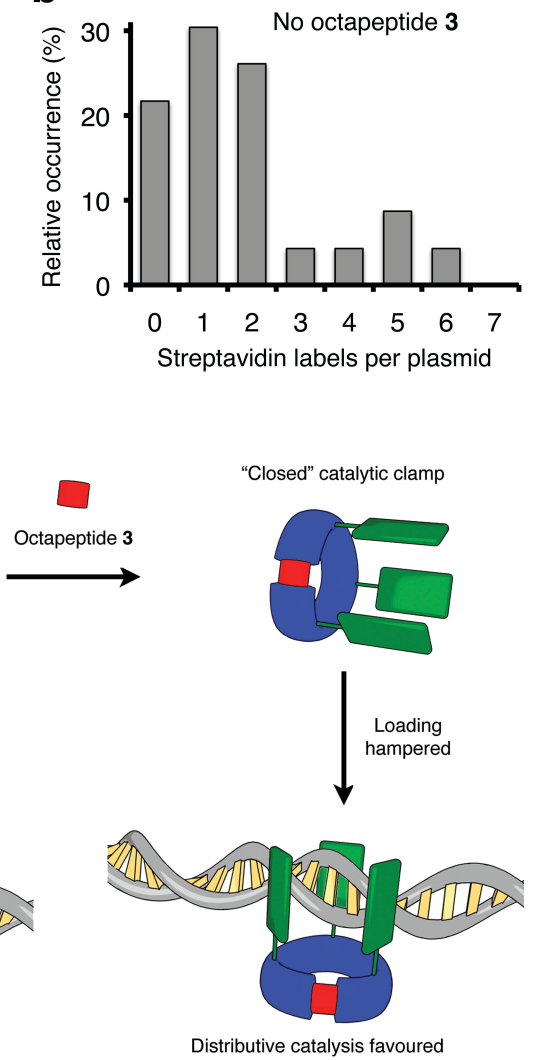

Figure 3 I Processive and distributive catalysis. a, Experiments in which the supercoiled plasmid was incubated with the catalytic clamp in the presence of $\mathrm{KHSO}_{5}$ (5 $\mu \mathrm{M})$ with or without octapeptide $3(10 \mu \mathrm{M})$ as indicated. The reaction was stopped after the indicated time (in minutes). $\mathrm{MW}=$ molecular weight marker (Hindlll digest); $\dagger$ indicates the running height of nicked, relaxed plasmids; $\ddagger$ indicates the running height of supercoiled plasmids; ${ }^{\circ}$ indicates the running height of linear DNA (faintly visible). b, Relative frequencies of oxidation reactions on individual plasmids after 5 minutes based on the streptavidin labelling analysis. For each encountered plasmid, the number of reactions was counted. No octapeptide was present. c, AFM images of plasmids oxidised by the catalytic clamp, with or without octapeptide $\mathbf{3}$ present as indicated. Without the peptide, clusters of streptavidin label can be found (top panel and expanded view in middle panel). These clusters never appear when the peptide is present (bottom panel). $\mathbf{d}$, Schematic 
representation of the influence of the octapeptide: spontaneous loading of the catalytic clamp can lead to processive oxidation. This is hampered by octapeptide $\mathbf{3}$, which closes the open subunit interface of the clamp. ${ }^{* * *}$ The text in this panel has been altered to become more balanced in its formulation ('hampered' instead of 'inhibited' and 'favoured' instead of 'only')
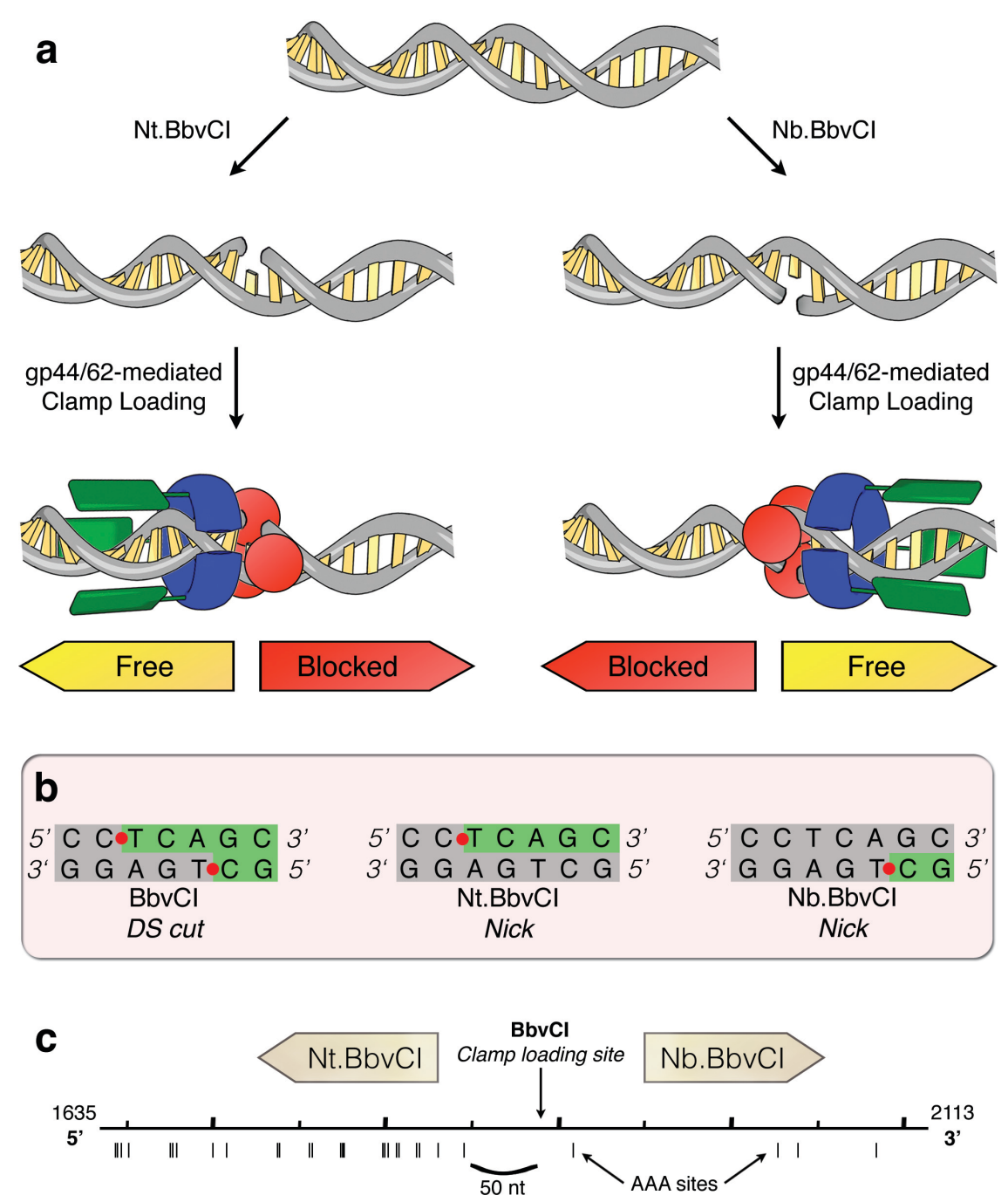

Figure 4 I Guiding the direction of the catalytic clamp. a, Schematic representation of the strategy followed: the clamp loader complex gp44/62 physically blocks one of the two directions in which the catalytic clamp can slide. When a nick is provided as a loading site, 
gp44/62 binds to its 3'-end. Therefore, nicks in opposite strands should direct the clamp in opposing directions. b, Recognition sequence and cut sites. The restriction endonuclease $\mathrm{BbvCl}$ produces sticky ends. Its variants (isoschizomers) $\mathrm{Nt} . \mathrm{BbvCl}$ and $\mathrm{Nb} . \mathrm{BbvCl}$ only cut once and thus produce nicks. ${ }^{29} \mathbf{c}$, Schematic representation showing a segment of the pGEM-derived plasmid (nucleotides 1635 to 2113 from the origin of replication). Potential oxidation sites are marked with black bars below the sequence. The $\mathrm{BbvCl}$ restriction site is indicated, and the arrows show the directions that are open to the catalytic clamp when the specified enzymes are used.
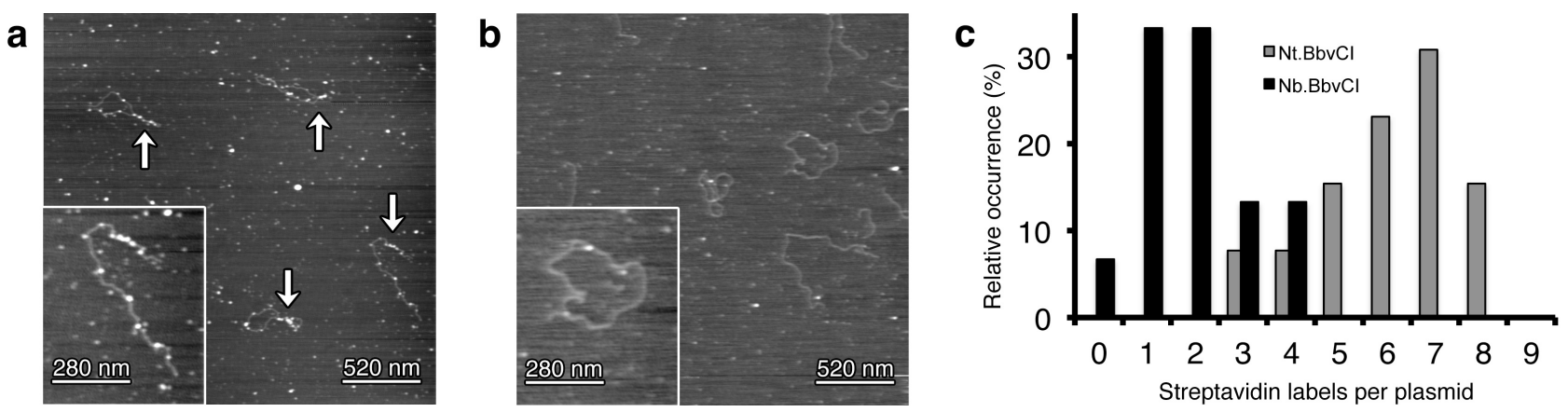

Figure 5 I AFM analysis of directed plasmid oxidation. a, AFM image of streptavidinlabelled plasmids after 5 minutes of oxidation by the catalytic clamp. The clamp was loaded by the gp44/62 clamp loader complex on a nick produced by Nt.BbvCl. Arrows point to regions with multiple streptavidin labels in a row. b, AFM image of streptavidinlabelled plasmids after oxidation by the catalytic clamp. The clamp was loaded by the gp44/62 clamp loader complex on a nick produced by $\mathrm{Nb.BbvCl}$. The lack of clustered streptavidin labels is apparent. c, Quantification of the amount of oxidation reactions on individual plasmids based on streptavidin labelling analysis. The relative frequency of the number of streptavidin labels is given for each plasmid. The catalytic clamp was loaded on 
nicks produced by either $\mathrm{Nb}$. $\mathrm{BbvCl}$ or $\mathrm{Nt} . \mathrm{BbvCl}$, and a significant difference between the resulting amount of oxidations was found (two-sample t-test: $p<0.0001$ ). 